\title{
Performance Evaluation and Quality Assurance Management During the Series Power Tests of LHC Main Lattice Magnets
}

\author{
A. Siemko and P. Pugnat
}

\begin{abstract}
Within the LHC project, a series production of superconducting dipoles and quadrupoles has recently been completed in industry and all magnets were cold tested at CERN. The main features of these magnets are: two-in-one structure, $56 \mathbf{~ m m}$ aperture, two layer coils wound from $15.1 \mathrm{~mm}$ wide $\mathrm{Nb}$-Ti cables, and all-polyimide insulation. This paper reviews the process of the power test quality assurance and performance evaluation, which was applied during the LHC magnet series tests. The main test results of magnets tested in both supercritical and superfluid helium, including the quench training, the conductor performance, the magnet protection efficiency and the electrical integrity are presented and discussed in terms of the design parameters and the requirements of the $\mathrm{LHC}$ machine.
\end{abstract}

Index Terms-Power tests, quality assurance, quench training, superconducting magnets.

\section{INTRODUCTION}

$\mathbf{T}$ HE Large Hadron Collider (LHC) at CERN [1], presently under construction and subsequent commissioning [2] is an accelerator with unprecedented complexity where the energy stored in magnets and beams exceeds other accelerators by at least one order of magnitude. To ensure as smooth as possible machine commissioning and safe start-up without being overwhelmed by major technical problems, the series power tests in operational cryogenic conditions, consisting of the individual thorough commissioning and qualification of all main superconducting magnets for the LHC ring were introduced. This activity started in early 2001 with the commissioning tests of the first 30 pre-series dipole magnets followed by the tests of over 1200 series dipoles. In the fall of 2003, the tests of the 360 so-called lattice short straight section magnets housing the main quadrupoles have commenced in parallel to the dipole magnets. Finally, in the fall of 2004, the tests of 114 special short straight section magnets housing the dispersion suppressor and matching section quadrupoles and a variety of corrector magnets were introduced. The series power tests of all LHC main lattice magnets lasted until February 2007 when the very last units were qualified for the installation in the accelerator tunnel.

An important effort went into the design and construction of the superconducting magnet test facility before main LHC series

Manuscript received August 29, 2007.

The authors are with the European Laboratory for Nuclear Research, CERN, Geneva CH-1211, Switzerland (e-mail: Andrzej.Siemko@cern.ch).

Color versions of one or more of the figures in this paper are available online at http://ieeexplore.ieee.org.

Digital Object Identifier 10.1109/TASC.2008.921904

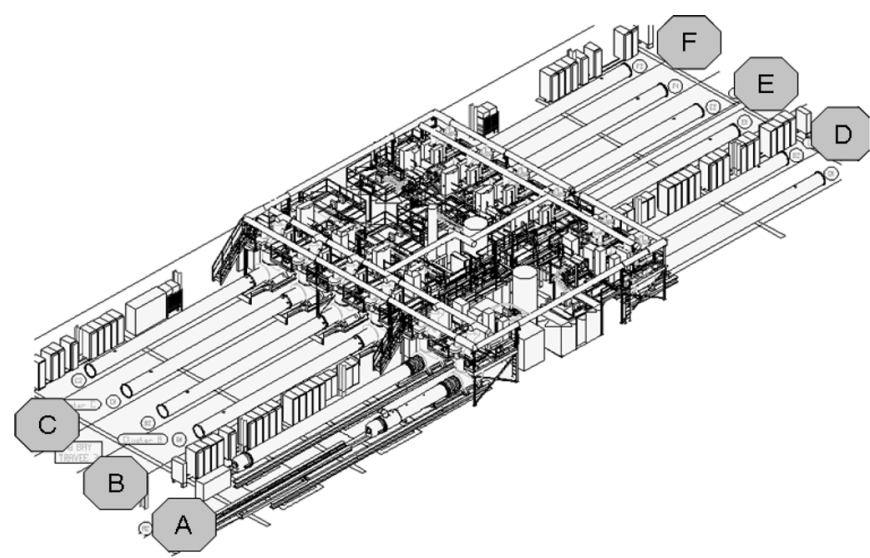

Fig. 1. Layout of the LHC superconducting magnet test plant consisting of 12 test benches grouped in 6 clusters (A to F).

power testing started. The organization, definition of detailed test scenarios, preparation of the tools to carry out the tests and measurements as well as to record and analyze the test results also received a lot of attention.

This paper discusses the experience with this approach, presents main results from the power tests of all LHC main magnets and describes some of the innovative tools and strategies which played crucial roles in the successful completion of the LHC magnet tests. Needless to say that the LHC superconducting magnet test program has profited from the previous projects [3]-[5].

\section{INFRASTRUCTURE FOR COLD TeSTS}

\section{A. Superconducting Magnet Test Plant}

Dedicated Superconducting Magnet Test Plant (SMTP), compatible with industrial production of over $1700 \mathrm{LHC}$ main magnets has been designed and constructed at CERN to accomplish the goal of testing all the magnets prior to their installation in the LHC tunnel. The test facility consists of 12 fully equipped test benches arranged in 6 clusters (see Fig. 1) which can run independently [6]. Each test bench is composed of a Cryogenics Feed Box (CFB) with its control system, a mechanical support structure and instrumentation racks containing the quench detection electronics, the magnet protection system with the quench heater power supplies, interlocks and also two independent data acquisition systems acquiring the data either at a high frequency range of 5 to $50 \mathrm{kHz}$ or at a low frequency range of 1 to $1000 \mathrm{~Hz}$. Racks with noise sensitive instrumentation, like electronics for magnetic measurements were separated 


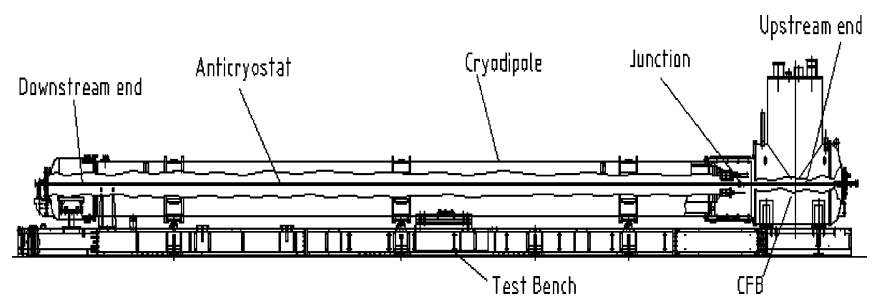

Fig. 2. Layout of a cold test bench with a cryo-dipole connected to its Cryogenic Feed Box (CFB). The overall length is equal to $19.6 \mathrm{~m}$.

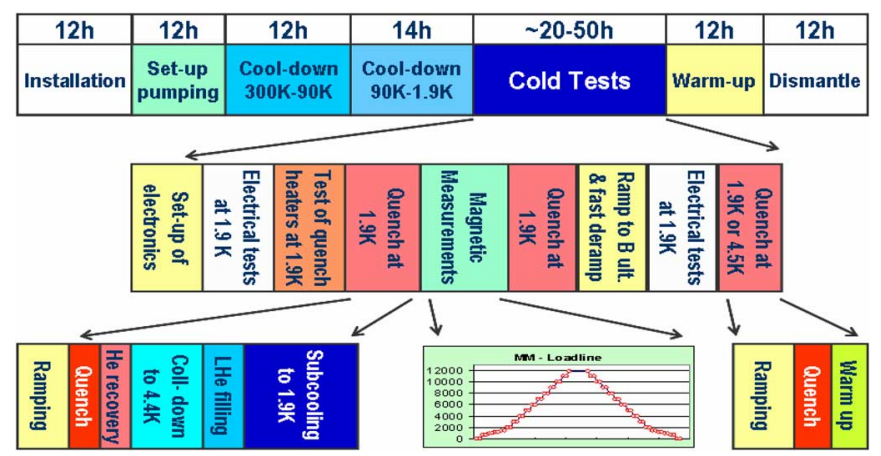

Fig. 3. Main phases of the standard test cycle for the LHC main dipoles.

from the power electronics. The two benches of each cluster share the main power converters as well as the instrumentation racks. To allow the commutation between the two test benches of one cluster, the common power converters (16 V/14 kA and $\pm 600 \mathrm{~A} / \pm 10 \mathrm{~V}$ ) and common electronic racks were connected with each of the two test benches passing through specially designed semi automatic commutation system, implemented in each cluster.

Cryogenic and electric feeding was done through the CFBs connected to one end of the magnet. A Magnet Return Box (MRB) closed the opposite end of the cryo-magnet under test. The functions of the CFB were to control the cool down and warm up of a cryo-magnet, to maintain a magnet cold mass in saturated liquid helium at $4.5 \mathrm{~K}$ or in pressurized superfluid helium at $1.9 \mathrm{~K}$ for magnetic measurements, power tests and quench training [7]. The CFBs were optimized to recover as much liquid helium as possible after a quench and to automatically cool-down the magnet again. They also contain all the current leads for the main coil and for the auxiliary corrector magnets.

As the magnetic measurement equipment only operates at ambient temperature, the beam screens were not mounted in the magnet apertures to permit insertion of the so-called "anticryostats", which allow the use of measuring shafts at room temperature for quench location and magnetic field measurements [8]. The layout of a dipole magnet installed on a bench is shown in Fig. 2. Construction of the SMTP was completed in spring 2004.

\section{B. Cold Test Phases}

Standard test sequences for the LHC main cryo-dipoles are shown in Fig. 3. Individual phases of the cold tests are briefly described in the following paragraphs.

1) Installation Phase: The cryo-magnets after their final preparation for the cold tests were transported by means of one of the dedicated transport vehicles to one of the test benches in SMTP. There, the magnet was first placed onto three support posts anchored to the test bench base structures. After a set of electrical reception tests, the magnet was aligned vertically and horizontally on the test bench. Typically, both ends of a magnet requiring magnetic measurements were aligned with a precision of a few tenth of a millimeter. All electric and hydraulic connections to the CFB unit, including anticryostats, were formed and followed by tests simulating magnet contraction during the cool down phase. The MRB, which closes the opposite end of the cryo-magnet, was anchored to the force retaining supports of the base structure. The test benches base structures were design to retain all mechanical forces appearing during the tests and to guarantee alignment and stability of a cryo-magnet throughout the test campaign. The installation of the thermal shield in the connection zone and closing of the vacuum vessel by means of a dedicated bellow completed the installation phase.

2) Setting-Up and Pumping Phase: When all electric, hydraulic and vacuum connections were completed and tested, three pumping purges between 1 bar and 20 mbar were executed and followed by an internal leak test using the leak detector integrated in the CFB. The leak signal was typically below $10^{-6} \mathrm{mbar} 1 / \mathrm{s}$ at a pressure of Gaseous Helium (GHe) in the magnet of 1.5 bars. In the next stage, the insulation vacuum of the CFB and magnet was pumped down to $10^{-3}$ mbar and globally leak tested.

3) Cool Down to $90 \mathrm{~K}$ : The cool down from 300 to $90 \mathrm{~K}$ was done by $\mathrm{GHe}$ pre-cooled with liquid nitrogen in a dedicated heat exchanger, which is not part of the CFB. The cold GHe entered in the system and passed through the magnet and the magnet thermal shields. Both flows were controlled such as to pass the maximum possible flow through the magnet cold mass $(\mathrm{CM})$ and at the same time to maintain the thermal shield at its nominal temperature range between 50 to $70 \mathrm{~K}$.

4) Cool Down to $4.2 \mathrm{~K}$ : When the magnet temperature reached $90 \mathrm{~K}$, the cooling was pursued by replacing in the same circuit, GHe with Liquid Helium (LHe). Whenever a CM was filled with saturated LHe at about 1.3 bars, the level in the phase separator and in all current lead vessels was set and kept at the required value. In this phase a magnet could be operated at $4.5 \mathrm{~K}$.

5) Cool Down to $1.9 \mathrm{~K}$ : Once a cold mass was filled with saturated liquid helium it could be cooled further to its operational temperature. For this purpose, the CFBs were equipped with a low-pressure circuit connected to a liquid/liquid heat exchanger, built in the magnet. Cold low pressure gas pre-cooled the LHe in a liquid/gas heat exchanger, which was pumped via a valve controlling the pressure. The maximum flow rate capacity of the pumping circuit was about $24 \mathrm{~g} / \mathrm{s}$ at 15 mbar.

6) Cold Tests-Powering the Magnets: In order to power the magnet coils, the CFBs were equipped with one pair of main $13 \mathrm{kA}$ current leads and two pairs of $1 \mathrm{kA}$ auxiliary current leads. The magnet coils were operating in pressurized superfluid helium at $1.9 \mathrm{~K}$ and 1 bar. All current leads were operating in saturated LHe at $4.5 \mathrm{~K}$. For this reason, so-called lambda plates physically separated the two helium circuits. The two coils that form the LHC two-in-one dipole magnet were powered in se- 


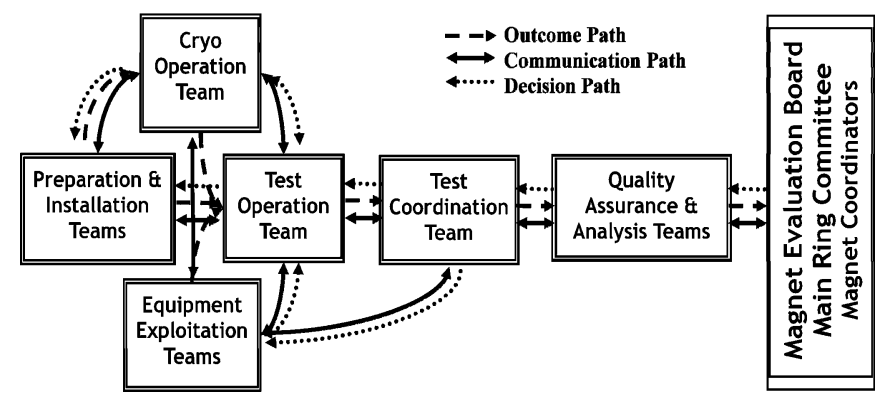

Fig. 4. The cold test organization and process flow chart.

ries using the main $13 \mathrm{kA}$ current leads. Auxiliary magnets, namely the sextupole, octupole and decapole correctors were power tested only for all pre-series cryo-dipoles and intermittently, during dedicated tests, for the series production magnets. The quadrupole coils, like dipole coils, were also powered in series making use of the main $13 \mathrm{kA}$ current leads. Unlike the corrector magnets integrated with the dipoles, the corrector magnets integrated with quadrupoles were all power tested. Powering of magnets on the test benches was performed up to the ultimate current level. For the main dipole and quadrupole magnets, quench tests were systematically carried out. During the quench tests, the liquid helium around the coil vaporizes and the pressure increases rapidly up to about 16 bars. A mixture of gas and liquid helium passed through a "quench" discharge valve into a decanter of about 400 liters volume. The gas could partially leave the CFB-magnet hydraulic circuit through the recovery line and/or the low-pressure line. The LHe remaining in the decanter was used for re-cooling the cold mass after a quench if required by the test program.

7) Warm-Up Phase: At the end of each measurement campaign, the magnets were quenched in order to vaporize most of the LHe. The heaters installed inside the decanter and in the phase separator boiled off the remaining LHe. Once it was evaporated, the magnet was warmed up to room temperature with a flow of warm GHe using the same hydraulic circuit as during the cooling phase.

8) Dismantling Phase: During this last phase of the test cycle, the cryo-magnets were disconnected from the cold test stands in inverse order with respect to that of the installation phase. The tested magnets were transported back to the assembly hall for the survey measurements and preparation for the storage.

The typical duration of the series magnet test cycle on the series test benches was of the order of 4 to 5 days and was comparable to the design value.

\section{Cold Test Organization}

The cold tests of the LHC superconducting magnets have required an important effort in the running and operation of a complex superconducting magnet test plant. The cold test organization implemented in the test facility involved many independent teams. The organization chart, showing the main teams and interactions among them, is presented in Fig. 4.

Round the clock operation concerned three teams: magnet connection/disconnection, cryogenic operation, and tests operation teams. The equipment exploitation teams were responsible for the test station hardware and software, its troubleshooting, and also the overall exploitation, maintenance and improvements of the test systems and infrastructures. These teams, working on-call basis supported the three operation teams. The engineers of the test coordination team provided the overall supervision and troubleshooting in case of problematic magnets and other nonstandard problems. In the immense effort of testing all LHC main magnets in cryogenic conditions within a strictly limited time frame, all these teams worked in close collaboration.

\section{Quality Assurance Plan for the COLD Tests}

\section{A. Quality Assurance Process and Main Quality Management Tools}

The high quality requirements for the performance of the LHC superconducting magnets demanded by the collider specifications necessitated setting up Quality Assurance Plan (QAP) for the series cold test with the aim of accepting only those magnets produced in industries which fully satisfy all of the tight LHC technical requirements and standards.

The cold test QAP referred to the existing LHC Project quality procedures that have been standardized and used within the organization. In particular, this concerns the so-called Manufacturing and Test Folder System (MTF) being an integral part of the Engineering Data Management System (EDMS) at CERN. The MTF was developed to acquire manufacturing and test data for the whole LHC Project [9], [10]. A wide range of data such as test results, non-conformity documents and other cold test related documentation have been stored in the MTF system. The MTF, which was created in close collaboration with the LHC Project Quality Assurance Working Group, implied, for the cold tests, the use of rules compliant with the LHC Project QAP [11], which had to be implemented within the cold test activities before using the MTF system. Important for cold tests part of the MTF is the so-called workflow tracking and handling the documentation belonging to different steps in the test process. For each individual step, information about results and possible nonconformities was stored and then used, by the cold test quality managers. The MTF was also used for the tracking and the maintenance management of cold test measuring systems and electronics.

The quality of the performed cold tests and measurements and their results was the focal point through the whole campaign of the series cold tests. Already from the first pre-series magnet tests, well defined test procedures were rigorously applied. All tested magnets had to fulfill respective acceptance criteria prior to their acceptance for the use in the collider.

\section{B. Automation of Series Tests and Measurements}

As anticipated already before the series tests start-up, the main quality issue was related to human errors in execution of repetitive tasks. In order to reduce this factor to the strict minimum, several measures were undertaken. First of all, an important effort was devoted to automate the tests and measurements, the data reduction and analysis. The testing process could be greatly accelerated and facilitated with the use of automation, which saved a lot of time and effort. The so-called Test Master 
[12] was the first application designed to automate the execution of series measurements in a single cluster. This application was responsible for launching sequences of tests and handling the applications to control all measurement devices necessary to acquire data, such as power converters, acquisition systems for quench tests or shafts and rotating units for magnetic measurements. However, the Test Master was neither capable to know if the necessary equipment was installed nor capable of generating the configuration files necessary for the applications. To set up every test, the measurement applications had to be configured taking into account the specific calibration parameters of the magnet and devices used. Due to the large number and diversity of configuration files needed to be prepared, there was a high risk of introducing errors when preparing manually these configurations. To cope with this important quality issue, an innovative tool called the Hardware Recognition System (HRS) [13], [14], based on a bar-code system linked to an Oracle database, has been developed. It allows the test operators to identify the test equipment and its corresponding calibration parameters as a function of the type of the test to be performed. The extracted data were used for automatic generation of the configuration files needed for the measurement applications. Within the framework of the HRS, a configuration database was created. This database stored scanning results of all equipment bar-codes, and allowed traceability of the equipment in combination with their parameter settings and use for the cold tests. The HRS system was a crucial component of completely automated measurement procedures, minimizing setting-up time and preventing human errors.

Also, the data reduction and analysis were highly automated. This was successful for most of the electrical tests and measurements. For this group of tests, the measuring systems were generating a go/no-go status after an analysis of the acquired data allowing the automatic test acceptance and permitting operators to execute the next step in the so-called "to-do-list". The quench tests were the second group of the most complex tests from the data reduction point of view, which were automated. The innovative Automatic Quench Analysis system (AQA) developed at CERN is capable to analyze and to judge the correctness of the quench process and magnet protection in case of all types of quenches performed routinely on all types of main magnets. After each performed quench, the AQA process resulted in the generation either of a conformance report or an alarm state, requiring further manual analysis of the data and expert judgment and the decision about the next step to be done. The AQA system also ensured a first validation of the quench results at the test operator level. The applied strategy for all data analysis applications was to store and preserve all raw data to allow a complete traceability of the test results as well as the possibility of an iterative analysis with improved software versions.

In addition, a set of web-based tools was introduced by the operation teams to aid the tests, in particular to automate the test results logging [15] and to ensure smooth interaction between the teams during the different stages of tests [16].

Unprecedented level of automation of the test and measurement systems in the LHC test facility, as compared to other test facilities of this type, allowed safe and efficient operation of the power tests by inexperienced operators, after relatively short training period [17].

\section{The Streamlined Cold Test Programs}

During the first international review of series tests of LHC lattice magnets held in July 2000 [18], the review board recommended to consider the cold series tests as mandatory to guaranty the accelerator reliability and performance. It specifically stressed the importance of testing all components related to magnet protection in conditions as close as possible to the machine operation. Reaching the required test efficiency was considered as a challenge in view of existing planning. The review board also strongly supported the decision to construct and fully equip 12 test benches for the cold tests.

To obtain a safe guaranty of the electrical integrity of the main magnets, it was considered necessary to quench each of them at least two times at or above the nominal current of $11850 \mathrm{~A}$. Only high current quenches provide conditions close to known failure modes of superconducting magnets with relevant Lorenz forces and voltages which develop in presence of gaseous helium. In addition, to assure the LHC operation at nominal energy, criteria based on the magnet quench performance were considered from the beginning of the elaboration of the cold test program.

The result-driven cold test program of Main Bending dipoles (MB) and Main Quadrupoles (MQ) was reviewed and streamlined in 2003. During two internal reviews, constraints coming from the LHC planning, the test duration and the capacity of the test station were analyzed [19], [20]. As a result, the quench performance evaluation was first based on a "two quench" criterion with a threshold at $12 \mathrm{kA}$. An extended test after a Thermal Cycle (TC) was executed for magnets that neither satisfied the "two quench" criterion nor reached $9 \mathrm{~T}$ after 8 quenches. In 2005, a "three quench" criterion was finally settled [21] with a threshold at $12.25 \mathrm{kA}$ and the same rule to perform additional training quenches after a TC. The test program also includes several electrical checks and measurements to ensure the continuity of each circuit, the proper insulation between circuits and between each circuit and the ground. The adopted strategy consists of performing electrical tests after each step that is judged critical, namely quench tests, cool down and warm-up phases.

\section{The Cold Test Quality Control}

To guarantee the effectiveness and thoroughness of the cold tests and the test results, three levels of quality control were introduced. The first validation of the test results and first level of the quality control were ensured directly by test operators. It is well known that an independent quality control is standard in the industry to ensure the quality of deliverables. This principle was also applied to the series tests of the LHC magnets at CERN.

The test facility equipment exploitation team made up of test and measurement experts, independent from the operation teams, was charged with the second level of cold test quality control. In this framework, the team members were working on a weekly rotation basis as the cold test Quality Managers (QM). The main responsibility of the QM team was to ensure the completeness and the conformity of all cold tests and measurement 
TABLE I

OVERVIEW OF THE NONCONFORMITIES DETECTED AT CERN ON 1246 CRYODIPOLES

\begin{tabular}{lcc}
\hline \hline NC Type & $\begin{array}{c}\text { Absolute Number } \\
\text { of NC }\end{array}$ & $\begin{array}{c}\text { Average Number of } \\
\text { NC by MB }\end{array}$ \\
\hline Mechanical & 1181 & 0.95 \\
Electrical & 583 & 0.47 \\
Quench Performance & 94 & 0.08 \\
Other & 275 & 0.22 \\
Total & $\mathbf{2 1 3 3}$ & $\mathbf{1 . 7 1}$ \\
\hline \hline
\end{tabular}

data and to make certain that all nonconformities were identified and all relevant documents recorded in the MTF system. The second objective was to follow the nonconformities, including the definition of the corrective actions for the problems encountered during the execution of the cold tests. Important part of this activity was special performance measurements and testing, carried out by experts to show not only whether tested magnet performance conforms to the specifications, but also to determine potential bottle-necks and improvements crucial for the performance.

The QM team, in collaboration with the cold test project engineers, was also responsible for the definition and maintenance of the documents used in the test process like the templates and checklists. The QM team managed as well the cold test related MTF quality records and their validation. The third quality control level was assured by the cold test project engineers who assessed the performance and the conformance to the technical requirements of each tested magnet. The cold test quality control process was accomplished by issuing a performance assent document.

As a final remark concerning the quality control of the cold tests of the LHC magnets, it is important to realize that the human errors, crucial for the magnet acceptance, were detected by all three levels of the quality control process.

\section{Main Results of The Cold Test}

The testing and qualification activities of the magnets were intended to verify their cryogenic, mechanical and electrical integrity, to qualify the performance of the magnet's protection systems and to verify magnets capability to reach required field level after limited number of training quenches. The cold magnetic measurements were performed on a fraction of the magnets in order to characterize the intended magnetic field. The cold tests were also the final stage in the acceptance of the magnets, based on their electrical and quench training performance and generally, meeting the full set of specification criteria.

\section{A. Encountered Nonconformities}

1) Case of Cryo-Dipoles: A total of about 2133 nonconformities (NC) encountered at CERN, concerning 1246 cryodipoles is listed in Table I as a function of their main characteristics. Around $90 \%$ of the electrical NC of Table I were discovered during the cold tests or preparatory phases on test benches.

The average number of each NC type by cryodipole was stable since the middle of the production. The proportion of

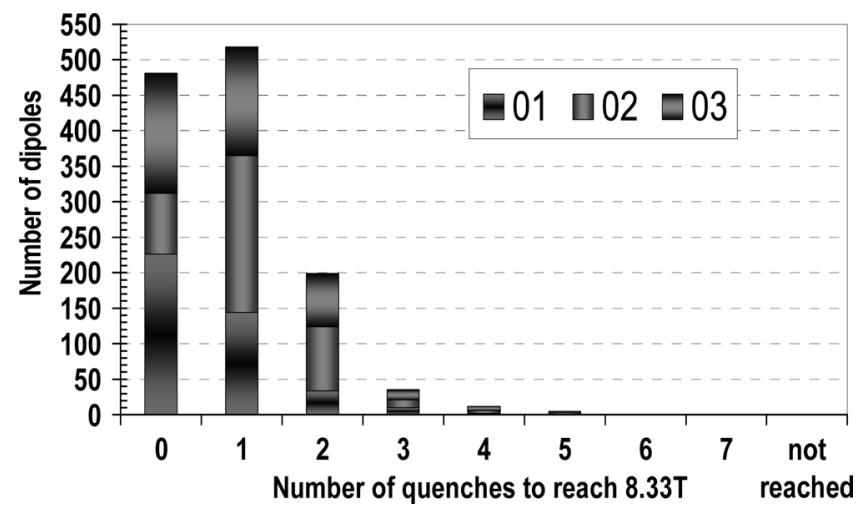

Fig. 5. Histogram after the first cool-down for $1252 \mathrm{MB}$ produced by the three manufacturers as a function of the number of training quenches.

MB with low quench performance which were submitted to a thermal cycle for extended tests was about $8 \%$ whereas only $\sim 1.1 \%$ were rejected for insufficient quench performance. In total 30 dipoles were rejected (2.4\%): 14 dipoles $(1.1 \%)$ due to unacceptable training performance and 16 dipoles $(1.3 \%)$ due to electrical nonconformities. Around $90 \%$ of the electrical NC of Table I was discovered during the cold tests or preparatory phases on the test benches. The cold tests allowed separating out magnets that would otherwise compromise the accelerator performance and permitted its projection [22].

2) Case of Short Straight Sections: The arc Short Straight Sections (SSS) are housing the MQ and various corrector magnets. The average number of electrical NC per SSS detected during cold tests or preparatory phases was around 0.76 , i.e., $\sim 1.5$ times larger than for the cryodipoles (cf. Table I). This comes from the larger number of electrical problems detected mostly because of the higher complexity of the electrical circuits in SSS cold masses with respect to the one of MB. In average, about $13 \%$ of MQ exhibited poor quench performance and were submitted to a thermal cycle for extended tests whereas only one was rejected.

\section{B. Training Quench Performance}

1) Training Quench Performance of Main Dipoles: The histogram of the cold tested main dipoles as a function of the number of training quenches required to reach the nominal field of the LHC is shown in Fig. 5. Before the thermal cycle (TC), about $38 \%$ of $\mathrm{MB}$ reached the nominal field of $8.33 \mathrm{~T}$ without training quench during their first powering. After a TC performed on about $9 \%$ of $\mathrm{MB}$, mostly due to weak quench performance, this proportion reached about $75 \%$.

2) Training Quench Performance of Main Quadrupoles: The histogram of the cold tested MQ as a function of the number of training quenches required to reach the nominal field gradient of $223 \mathrm{~T} / \mathrm{m}$ is shown in Fig. 6. Before the thermal cycle, about 56\% of MQ reached the nominal field gradient during their first powering without quench. After a TC performed on only a limited number of MQ, this proportion reached about $60 \%$. To reach the LHC nominal energy level of $7 \mathrm{TeV}$ will imply to cope with training quenches of MBs, MQs as well as of other superconducting magnets. The expected quench performance of the main 


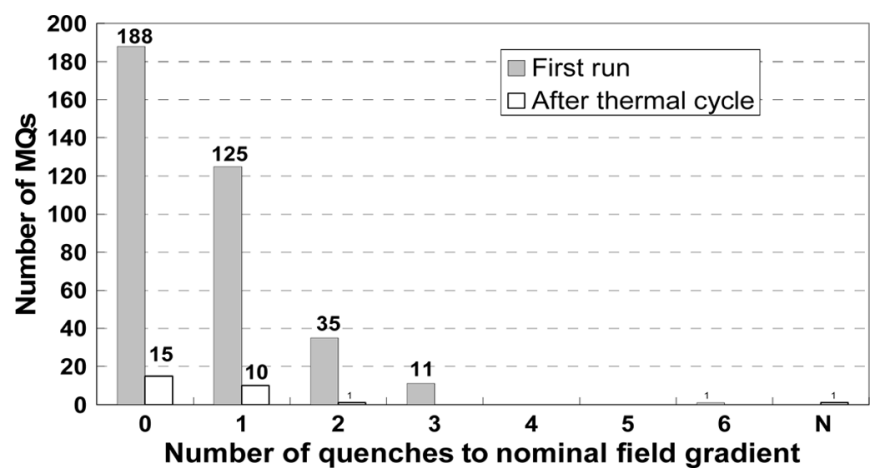

Fig. 6. Histogram after the first cool-down for $360 \mathrm{MQ}$ as a function of the number of training quenches.

dipoles and the main quadrupoles during their first powering cycles in the LHC machine is discussed in details in [22].

\section{CONCLUSION}

The cold tests of all LHC superconducting magnets were successfully completed within a tight schedule and had closely followed the magnet production in European industries. Unprecedented level of automation of the test and measurement systems allowed safe and efficient operation of the power test and measurements by inexperienced test operators, working on a one-year rotational basis. High reliability of the infrastructure and equipment, which for the whole test facility over 4 years exceeded $98 \%$ of the availability of the 12 test benches for the operation, has played a crucial role in the successful completion of magnet tests. The overall test results confirmed the need of testing systematically all the LHC magnets under cryogenic conditions, prior to their installation in the accelerator tunnel. The quality assurance plan and strict quality control implemented during the LHC magnet tests allowed to separate out magnet units that would otherwise compromise the accelerator commissioning and its performance.

\section{ACKNOWLEDGMENT}

The authors would like to thank all members of the SMTP teams for the production and the validation of the data, $\mathrm{N}$. Smirnov and M. Pojer for regular update of the statistical results of main superconducting magnets and D. Tommasini for providing the data of Table I.

\section{REFERENCES}

[1] The LHC Study Group, The Large Hadron Collider Design Report CERN-2004-003, Jun. 4, 2004, vol. I.

[2] R. Saban et al., "The commissioning of the LHC technical systems," presented at the PAC 2007, , unpublished.

[3] S. Wolff, The Superconducting Magnet System for HERA, C. Marinucci and P. Waymuth, Eds. Zurich: SIN, 1995.

[4] D. G. Warner and D. L. Bever, SSCL Magnet Systems Quality Program Implementation for Laboratory and Industry SSCL-PREPRINT-035, Mar. 1992.

[5] P. Wanderer et al., The RHIC Magnet System NIM A 499, 2003, pp. 280-315.

[6] A. Siemko et al., "Quench performance and field quality of the LHC preseries superconducting dipoles," IEEE Trans. Appl. Supercond., vol. 14, no. 2, pp. 165-168, 2004.

[7] L. Bottura et al., "The magnet evaluation board," in CERN LHC Project Workshop-“Chamonix XIV", Feb. 2005.

[8] J. Billan et al., "Twin rotating coils for cold magnetic measurements of $15 \mathrm{~m}$ long LHC dipoles," IEEE Trans. Appl. Supercond., vol. 10, no. 1, pp. 1422-1426, Mar. 2000

[9] T. Pettersson et al., "The CERN EDMS-Engineering and equipment data management system," in EPAC, Paris, Jun. 2002.

[10] P. Martel et al., "Manufacturing and test folder: MTF," in EPAC, Paris, Jun. 2002.

[11] P. Faugeras, Quality Assurance Policy and Project Organisation CERN-EDMS Id 103544.

[12] A. Rijllart et al., "Orchestrating the measurements of the twelve magnet test benches," in Proceedings EPAC 2002, Paris, France.

[13] A. Raimondo et al., "The hardware recognition system for the SM18 LHC cryomagnet test benches," in Proceedings of ICALEPCS 2003, Gyeongju, Korea, Oct. 13-17, 2003.

[14] T. Ladzinski et al., "The hardware recognition system for the SM18 LHC cryomagnet test benches," in Proceedings of ICALEPCS 2003, Gyeongju, Korea, Oct. 13-17, 2003.

[15] G. H. Hemelsoet et al., "Cryogenic magnet tests for the LHC: Process operation using web-based tools and facilities," in Proc. ICALEPCS 2005, Geneva, Oct. 2005.

[16] A. Tovar-Gonzalez et al., "Automatic management systems for the operation of the cryogenic test facilities for LHC series superconducting magnets," in Proc. CEC-ICMC, Keystone, CO, Aug. 2005.

[17] V. Chohan et al., "Operation for LHC cryomagnet tests: Concerns, challenges \& successful collaboration," presented at the Asian Particle Accelerator Conference (APAC 2007), Indore, India, unpublished.

[18] L. Walckiers et al., Review at CERN of Series Tests of LHC Lattice Magnets: Summary of the Recommendations of the Board Doc LHCMTA, Sep. 21, 2000, unpublished.

[19] Review of Reception Tests of LHC Dipole Cryomagnets CERN-EDMS Id 399278, unpublished, Jul. 2003.

[20] Review of Reception Tests of LHC Cryomagnets CERN-EDMS Id 427483, unpublished, Dec. 2003.

[21] Minutes of the CERN Internal Meeting MARIC 2005-112, unpublished.

[22] P. Pugnat and A. Siemko, "Review of quench performance of LHC main superconducting magnets," IEEE Trans. Appl. Supercond., vol. 17, no. 2, pp. 1091-1096, 2007. 